\title{
Hydrogen-free SiCN Films Obtained by Electron Cyclotron Resonance Plasma
}

A Study of Composition, Optical, and Luminescent Properties

\author{
M. J. Hernández, ${ }^{\text {a }}$ M. Cervera, ${ }^{\text {a J. Piqueras, }}{ }^{a}$ M. A. González,,${ }^{b}$ M. Avella, ${ }^{b}$
} T. del Caño, ${ }^{\mathrm{b}}$ and J. Jiménez ${ }^{\mathrm{b}}$

${ }^{a}$ Laboratorio de Microelectrónica, Universidad Autónoma de Madrid, Cantoblanco, 28049 Madrid, Spain

${ }^{b}$ Física de la Materia Condensada, ETSII, Universidad de Valladolid, 47011 Valladolid, Spain

Electron cyclotron resonance plasma enhanced chemical vapor deposition of hydrogen-free SiCN films has been studied. Infrared and Raman spectroscopies were used for chemical characterization of deposited films, showing $\mathrm{Si}-\mathrm{N}, \mathrm{Si}-\mathrm{C}, \mathrm{Si}-\mathrm{Si}$, and $\mathrm{C}-\mathrm{N}$ bonds in composition. Optical responses of the films between 1.5 and $4.5 \mathrm{eV}$ were obtained by spectroscopic ellipsometry. Cathodoluminescence of the films shows mainly a broad band of emission at around $2.3 \mathrm{eV}$ together with overlapped contributions at higher energies depending on the composition. Surface morphology and roughness has been investigated by atomic force microscopy. (c) 2007 The Electrochemical Society. [DOI: 10.1149/1.2472559] All rights reserved.

Manuscript submitted May 25, 2006; revised manuscript received December 18, 2006. Available electronically February 22,2007

In the last decade, there has been an increasing number of studies on SiCN compound synthesis, stimulated by the excellent mechanical and chemical properties of hardness and corrosion resistance of the amorphous material. ${ }^{1,2}$ A number of different techniques, such as ion implantation, ${ }^{3,4}$ chemical vapor deposition, ${ }^{5-16}$ or sputtering, ${ }^{17}$ among others, have been employed to obtain this material, since Gómez et al. suggested the possible miscibility of $\beta-\mathrm{Si}_{3} \mathrm{~N}_{4}$ and the hypothetical one, $\beta-\mathrm{C}_{3} \mathrm{~N}_{4} \cdot{ }^{18}$ The optical properties of $\mathrm{SiCN}$ suggest the behavior as a wide-bandgap semiconductor in the case of the crystalline phase, with potential applications for blue or ultraviolet optoelectronic devices. ${ }^{6,15}$ The variety of silicon carbonitride compositions obtained with any of these techniques allows one to think of these materials as tunable bandgap ones for applications in flat panel displays. Furthermore, $\mathrm{SiCN}$ nanorods exhibit promising characteristics as field emitters. ${ }^{9,19}$

More recently, with the introduction of new synthesis methods, other innovative applications have been described for this family of compounds, such as its feasibility in the fabrication of microelectrical mechanical systems (MEMS) working in high-temperature environments. ${ }^{20,21}$

With the continuous shrinking size in integrated circuits technology there is a continuous effort in the search for materials with low dielectric constant for metal layer isolation. Further, due to its low dielectric constant, hydrogenated silicon carbonitrides prevent $\mathrm{Cu}$ diffusion in between the metallization layers. ${ }^{22}$ The hydrogen incorporation in amorphous $\mathrm{SiCN}$ films results in a degradation of the mechanical properties of these films. ${ }^{8}$

In this work, we report the synthesis of amorphous hydrogen-free $\mathrm{SiCN}$ films by electron cyclotron resonance plasma at $850^{\circ} \mathrm{C}$. Their composition and bonding structure have been studied by Fourier transform infrared (FTIR) and Raman spectroscopy. The surface morphology has also been studied by atomic force microscopy. The dielectric constant for different layer compositions has been obtained by spectroscopic ellipsometry and their potential optoelectronic applications explored by cathodoluminescence measurements.

\section{Experimental}

SiCN films were deposited on float zone (FZ) silicon wafers, polished on both sides, at $850^{\circ} \mathrm{C}$ in an electron cyclotron resonance plasma (ECR) reactor. The substrate holder was radiatively heated with a tungsten filament lamp and the temperature, $850^{\circ} \mathrm{C}$, monitored with a pyrometer across a quartz window. The base pressure of the system was $10^{-7}$ Torr. Low oxygen content FZ silicon wafers with a resistivity higher than $100 \Omega \mathrm{cm}$ were used as substrates. Before deposition, silicon substrates were chemically etched with a

ž-mail: mrebollo@eis.uva.es
HF buffer solution. Gas mixtures of $\mathrm{Ar}, \mathrm{CH}_{4}, \mathrm{~N}_{2}$, and $\mathrm{SiH}_{4}$ were employed in the deposition processes; the precursor gas flows were controlled by mass flow meters in such a way that a wide range of $\mathrm{C} / \mathrm{N}$ and $\mathrm{N} / \mathrm{Si}$ atomic ratios could be attained, while maintaining the $\mathrm{C} / \mathrm{Si}$ flow ratio at 2.8 or 3.6 (series A and $\mathrm{B}$, respectively). Ar was introduced with the aim of attaining stable plasma, whatever the precursor's gas flow. The process time and the $\mathrm{SiH}_{4}$ flow were adjusted to achieve SiCN films with thickness around $850 \AA$, measured using a Dektak profilometer. This value assures the absence of interferential oscillations in the spectroscopic ellipsometry (SE) spectra associated with the thickness, which could cause a poor determination of the composition from the spectra fittings. The work pressure was kept in between 1 and 4 mTorr. The microwave power was fixed at $1000 \mathrm{~W}$ for all the processes. The chemical bonds present in the films were investigated by FTIR and Raman spectroscopy. FTIR spectra were obtained using a Bruker IFS-66v spectrometer with a spectral resolution of $6 \mathrm{~cm}^{-1}$. The Raman spectra were recorded in backscattering geometry using a Jobin-Yvon HR800UV Raman spectrometer. The excitation was done with different laser lines; however, the best results were obtained with UV excitation from a He-Cd laser $(325 \mathrm{~nm})$, and we refer to these spectra henceforth. The scattered light was dispersed by the spectrometer and collected by a liquid nitrogen cooled charge-coupled device (CCD) detector. SE measurements between 1.5 and $4.5 \mathrm{eV}$ were acquired at $70^{\circ}$ angle of incidence with a Jobin-Yvon Uvisel ellipsometer.

Luminescence spectra of the films were acquired by cathodoluminescence (CL) using a XiCLOne system from Gatan attached to a JEOL 820 scanning electron microscope (SEM), typically using $10 \mathrm{kV}$ acceleration voltage and $2 \mathrm{nA}$ beam current. Sample surface morphology was studied with a Topometrix atomic force microscope (AFM).

\section{Results}

Infrared characterization.- The IR spectra were corrected with the transmittance of a bare silicon wafer. Transmission spectra of the samples in the region of interest are plotted in Fig. 1 for the sample series $\mathrm{A}(\mathrm{C} / \mathrm{Si}=2.8)$. The spectra of the sample series $\mathrm{B}(\mathrm{C} / \mathrm{Si}$ = 3.6) were similar to those of sample series A; therefore, they are not shown here. Characteristic peak positions of $\mathrm{SiC}\left(796 \mathrm{~cm}^{-1}\right)^{23}$ and $\mathrm{Si}_{3} \mathrm{~N}_{4}\left(846 \mathrm{~cm}^{-1}\right){ }^{24}$ are indicated, for reference, by straight lines in the figure. The $\mathrm{C} / \mathrm{N}$ ratio values of the deposition processes are indicated for each FTIR curve. No bands associated with $\mathrm{H}$ bonds, usually at 2000-2100 $(\mathrm{Si}-\mathrm{H}), 3000(\mathrm{C}-\mathrm{H})$, and $3380 \mathrm{~cm}^{-1}$ $(\mathrm{N}-\mathrm{H})$ (not shown in the figure), were observed for any sample, as opposed to the spectra obtained in SiCN films deposited at lower temperatures $\left(400-500^{\circ} \mathrm{C}\right),{ }^{7}$ for which $\mathrm{C}-\mathrm{H}_{n}$ and $\mathrm{N}-\mathrm{H}$ bonds were detected. Neither double and triple $\mathrm{C}-\mathrm{C}$ nor $\mathrm{C}-\mathrm{N}$ bonds were de- 


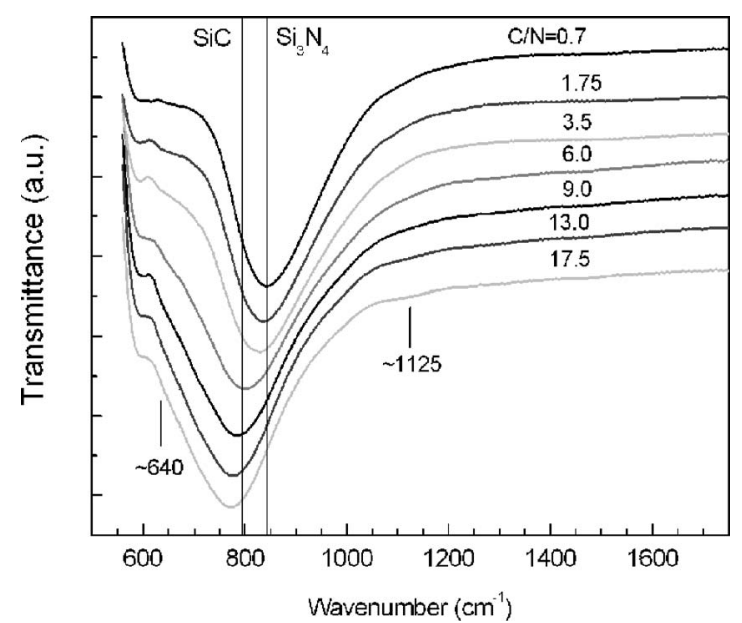

Figure 1. FTIR spectra of films deposited with ratio $\mathrm{C} / \mathrm{Si}=2.8$ for different $\mathrm{C} / \mathrm{N}$ ratios.

tected, although in the first case it could be due to the IR inactivity of the graphitic bonds. ${ }^{25}$ The main absorption band shifts from 770 to $843 \mathrm{~cm}^{-1}$, while the full width at half maximum (FWHM) becomes narrower (from 240 to $208 \mathrm{~cm}^{-1}$ ) for decreasing $\mathrm{C} / \mathrm{N}$ ratio. The measured FWHM values are larger than those obtained for the binary compounds, $\mathrm{Si}_{3} \mathrm{~N}_{4}$ and $\mathrm{SiC}$, around 200 and $90 \mathrm{~cm}^{-1}$, respectively (not shown here), deposited under equivalent experimental conditions. The bands peaking between 796 and $846 \mathrm{~cm}^{-1}$ can be related to the vibrational modes associated with $\mathrm{Si}-\mathrm{N}$ (exhibiting modes at 1021, 947, 838, and $800 \mathrm{~cm}^{-1}$ ) ${ }^{24}$ and with Si-C $\left(796 \mathrm{~cm}^{-1}\right)$ bonds. ${ }^{23}$ An additional shoulder contribution is observed at approximately $640 \mathrm{~cm}^{-1}$, more defined for the samples with the higher $\mathrm{C} / \mathrm{N}$ ratios. This band might be tentatively related to $\mathrm{Si}-\mathrm{H}_{2}$ and $\mathrm{Si}-\mathrm{H}$ wagging bands, typically observed in porous silicon at $622 \mathrm{~cm}^{-1} ;^{26}$ however, the nonobservation of the corresponding stretching bands of these bonds around $2100 \mathrm{~cm}^{-1}$ led us to disregard this assignation. Finally, for the same samples, a weak band at $1125 \mathrm{~cm}^{-1}$ of uncertain origin was detected.

Raman spectroscopy.- Figure 2 presents the Raman spectra of the sample series A $(\mathrm{C} / \mathrm{Si}=2.8)$. The spectra of the sample series $\mathrm{B}$ $(\mathrm{C} / \mathrm{Si}=3.6)$ were similar to those of series $\mathrm{A}$; therefore, we will focus later on the spectra of sample series A. One can separate the spectra into three spectral ranges. First, in the low-frequency region,

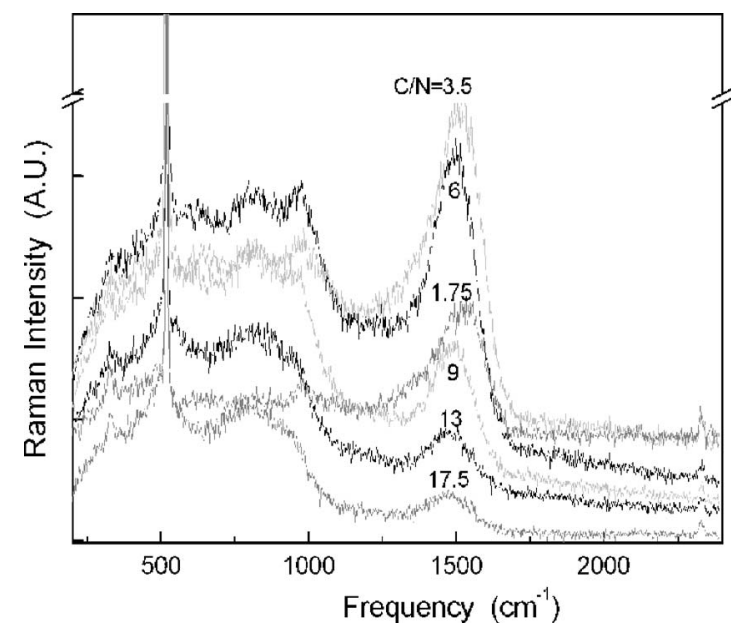

Figure 2. Raman spectra of samples, excited at $325 \mathrm{~nm}$ laser line, corresponding to $\mathrm{C} / \mathrm{Si}=2.8$ series for different $\mathrm{C} / \mathrm{N}$ ratios. two bands peaking at 300 and $520 \mathrm{~cm}^{-1}$ are observed. They arise from the Si substrate and are not relevant to our analysis; they correspond to the second and first-order Raman bands of crystalline $\mathrm{Si}$, respectively. Besides, a broad band located at $640 \mathrm{~cm}^{-1}$ is observed for the intermediate $\mathrm{C} / \mathrm{N}$ atomic flow ratios. Second, we can distinguish the spectral interval from 700 to $1000 \mathrm{~cm}^{-1}$. In this range one can observe two main broad bands; the first one (labeled I1) is observed at $790 \mathrm{~cm}^{-1}$, and it can be assigned to the transverse optical (TO) mode of cubic SiC. ${ }^{27}$ The second band (labeled I2), observed at $980 \mathrm{~cm}^{-1}$, can be associated with different origins: e.g., $\mathrm{Si}-\mathrm{N}$ bonds, ${ }^{28,29} \mathrm{Si}-\mathrm{C}$ longitudinal optical (LO) mode ${ }^{27}$ or the second-order spectra of the Si substrate, and will be discussed later.

Finally, the third spectral region, above $1000 \mathrm{~cm}^{-1}$, is characterized by a broad band around $1540 \mathrm{~cm}^{-1}$ with a well-defined shoulder in the low-frequency side, around $1450 \mathrm{~cm}^{-1}$. These bands, G $\left(1580-1600 \mathrm{~cm}^{-1}\right)$ and $\mathrm{D}\left(1360 \mathrm{~cm}^{-1}\right)$, can be related to the characteristic bands of carbon materials, disordered graphite, a-C, or diamondlike carbon. In the literature $\mathrm{G}$ is commonly assigned to the $\mathrm{E}_{2 \mathrm{~g}} \mathrm{C}-\mathrm{C}\left(\mathrm{sp}^{2}\right.$-bonded) stretching mode of single-crystal graphite, whereas the $\mathrm{D}$ band is attributed to the $\mathrm{A}_{1 \mathrm{~g}}$ stretching mode on the crystal boundaries of polycrystalline graphite, and appears when the long-range order of the graphite lattice is disrupted. ${ }^{30,31}$ Both peaks dominate, varying the intensity, position and width, the Raman spectra of nanocrystalline and amorphous carbons. ${ }^{32}$ Further, both $G$ and $\mathrm{D}$ bands are also usually observed in Raman spectra of a-CN films; ${ }^{33-35}$ however, $\mathrm{C}-\mathrm{N}$ and $\mathrm{C}-\mathrm{C}$ modes lie in a similar wavenumber range and thus are difficult to separate from each other. ${ }^{36}$ Often these bands broaden and overlap each other, ${ }^{37}$ which matches our observation. The peak position of both bands can provide useful information about the material properties, which will be assigned in the Discussion section.

Spectroscopic ellipsometry.- The pseudo-dielectric constant of the samples was acquired by SE measurements in the spectral range between 1.5 and $4.5 \mathrm{eV}$. The spectra were modeled on the basis of an air-film-substrate structure, the optical constants of the silicon substrate being known. Due to the lack of standard optical data for $\mathrm{SiCN}$ compounds, the films have been modeled in terms of a mixture of $\mathrm{Si}_{3} \mathrm{~N}_{4}$ and $\mathrm{SiC}$. The effective medium theories relate the dielectric response of an inhomogeneous material with clusters of dimensions smaller than the probe wavelength to its microstructure. ${ }^{38}$ The smoothness of the main band detected in FTIR spectra, $\approx 800 \mathrm{~cm}^{-1}$, suggests the existence of structural units on the scale of several angstroms, always smaller than the UV-visible probe wavelength; this is confirmed by the broad Raman bands, which account for reduced phonon correlation lengths due to translational symmetry breakdown.

Also, in most samples small crystalline-silicon (c-Si) fractions were considered to include the contribution of the $\mathrm{Si}$ atomic clusters (the $\mathrm{Si}$ atoms substituting for $\mathrm{C}$ in $\mathrm{Si}-\mathrm{C}-\mathrm{Si}$ sequences). The volume fractions of each phase and the thickness of the films were obtained for most of the samples making use of the Bruggeman effective medium approximation (EMA). ${ }^{39}$ Samples, either $\mathrm{SiC}$ rich or $\mathrm{Si}_{3} \mathrm{~N}_{4}$ rich, were also reliably modeled by the Maxwell Garnett (MG) model, as expected from the theoretical analysis. ${ }^{39}$ The inclusion of a graphitic phase in the fitting processes did not provide satisfactory results, which is in apparent contradiction with the Raman results that revealed the presence of disordered graphitic phases. This suggests that the Raman bands could be due to $\mathrm{CN}$ bonds rather than to $\mathrm{C}-\mathrm{C}$ bonds, even if the IR data could not confirm this assertion.

The $\mathrm{Si}_{3} \mathrm{~N}_{4}$ and $\mathrm{SiC}$ percentages were obtained by fitting the measured spectra, allowing the thickness value to vary in a very close range around the profilometric value. The fitted thicknesses of the films ranged from 830 to $910 \AA$. The volume fractions of $\mathrm{SiC}$ and $\mathrm{Si}_{3} \mathrm{~N}_{4}$ vs the N/Si ratio with the $\mathrm{C} / \mathrm{Si}$ ratio as a parameter are shown in Fig. 3. The samples with the higher c-Si percentage are those with the more pronounced $\sim 640 \mathrm{~cm}^{-1}$ contribution to the FTIR spectra. This fact suggests that the contribution of the Si-Si bonds to the $\mathrm{UV}$-visible spectrum is similar to the contribution obtained for a c-Si 


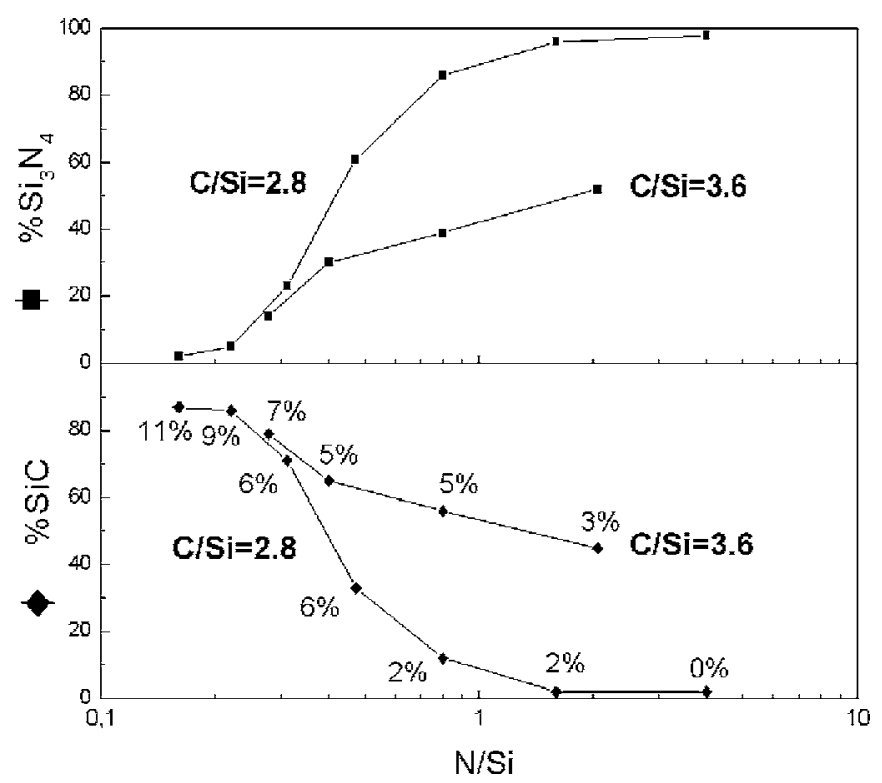

Figure 3. Percentages of composition of $\mathrm{SiCN}$ samples from fittings of the $\mathrm{SE}$ spectra. Films are modeled according to the EMA model, with $\mathrm{Si}_{3} \mathrm{~N}_{4}$ (top), $\mathrm{SiC}$ (bottom), and c-Si (added values to the low curves) phases.

phase, which suggests the formation of small clusters of $\mathrm{Si}$ atoms when $\mathrm{Si}$ replaces $\mathrm{C}$. From the same figure a higher incorporation efficiency of $\mathrm{N}$ than $\mathrm{C}$ to the films is deduced from the two series of samples. This may be due to the higher stability of the $\mathrm{Si}-\mathrm{N}$ bond than the $\mathrm{Si}-\mathrm{C}$ one, for which the bond energies are 3.45 and $3.21 \mathrm{eV}$, respectively.

The dielectric responses calculated by the effective medium model with values obtained from the best fittings are represented for some of the samples of series $\mathrm{A}(\mathrm{C} / \mathrm{Si}=2.8)$ as a function of the energy in Fig. 4 (real and imaginary parts). In the same figure, the dielectric responses of $\mathrm{Si}_{3} \mathrm{~N}_{4}$ and $\mathrm{SiC}$ have been added for reference. For the samples with the higher percentages of silicon $(\mathrm{C} / \mathrm{N}$ $>$ 6.0) the dielectric response values are higher than those of $\mathrm{SiC}$; simultaneously, peaks related to the E1 and E2 silicon critical points, near 3.4 and $4.2 \mathrm{eV}$, become apparent. ${ }^{39}$ Films with dielectric func-

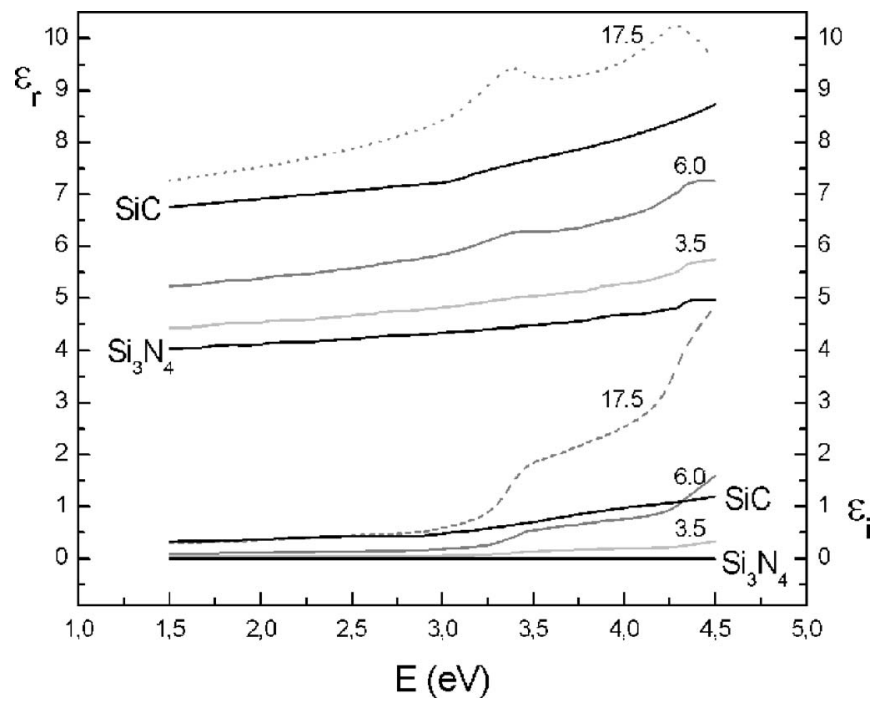

Figure 4. Dielectric response of representative $\mathrm{SiCN}$ films with $\mathrm{C} / \mathrm{Si}=2.8$ in accordance with the EMA model. (Upper curves, $\varepsilon_{r}$; lower curves, $\varepsilon_{i}$ ). $\mathrm{C} / \mathrm{N}$ ratio values have been added on the curves.

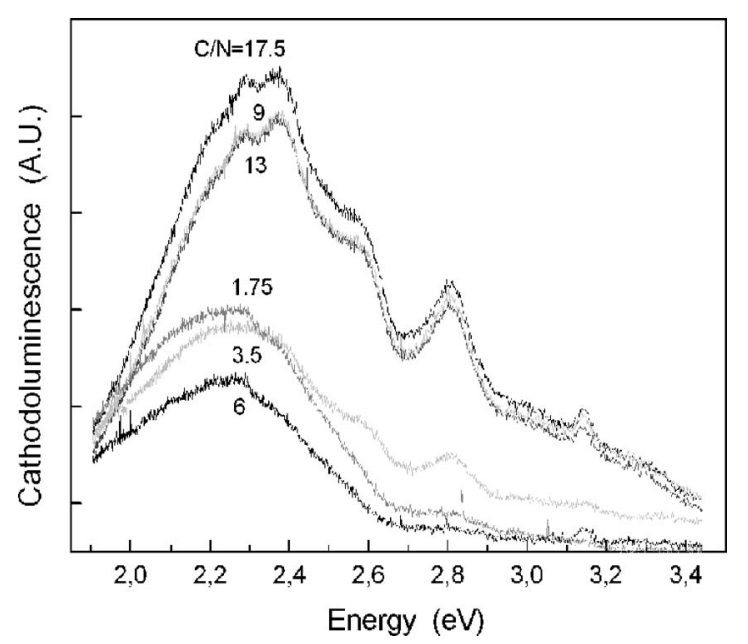

Figure 5. CL spectra of samples, excited at liquid nitrogen temperature, corresponding to $\mathrm{C} / \mathrm{Si}=2.8$ series.

tion in between those of $\mathrm{Si}_{3} \mathrm{~N}_{4}$ and $\mathrm{SiC}$ were obtained using higher $\mathrm{N} / \mathrm{Si}$ ratios. Comparing these curves with the dispersion curves of Gómez et al. ${ }^{18}$ for samples obtained with no intentional heating, a higher optical density of $\mathrm{SiCN}$ films is obtained in this case, related to the absence of hydrogen bonds.

Cathodoluminescence.-Figure 5 shows the CL spectra of sample series A $(\mathrm{C} / \mathrm{N}=2.8)$ obtained at $80 \mathrm{~K}$. The spectra consist of several broad bands, with several overlapped peaks at 3.14, 2.8, $2.57,2.38,2.27 \mathrm{eV}$ and a shoulder at $2.17 \mathrm{eV}$. For the samples with low $\mathrm{SiC}$ content and high $\mathrm{Si}_{3} \mathrm{~N}_{4}$ concentration, as deduced from the ellipsometric fittings, the CL intensity decreases, whereas the complex CL band is replaced by a structureless band peaking at $2.26 \mathrm{eV}$. The spectral features of the luminescence emission are strongly related to the composition and structure of the films; in particular, the cluster structure and size should have a great influence on the luminescence emission. ${ }^{40-42}$ The higher intensity of the CL bands and their corresponding shift to the high energies for samples with higher $\mathrm{SiC}$ content might be related to several causes: an increase of the probability of radiative recombination due to the presence of $\mathrm{SiC}$ clusters, the decrease of nonradiative recombination through defects, ${ }^{41}$ and the reduction of the cluster diameter with the increasing of [C] observed in Raman measurements. ${ }^{32}$ Further work is required to understand the origin of the peaks superposed to the broad band in $\mathrm{SiC}$ rich samples. These bands are narrower, suggesting the presence of relatively ordered clusters.

Atomic force microscopy.- The surface morphology of the samples was obtained by AFM. Figure 6 shows three-dimensional scans of an area of $1.1 \times 1.1 \mu \mathrm{m}$ of two samples. Figure 6a corresponds to a sample of series A, and Fig. $6 \mathrm{~b}$ to a sample of series B; both of them have been performed with $\mathrm{N} / \mathrm{Si}=0.8$ (see Fig. 3). The surface morphology was found to depend on $[\mathrm{N}]$ and $[\mathrm{C}]$. The rms roughness is similar in both samples (1.54 nm in Fig. 6a and $1.16 \mathrm{~nm}$ in Fig. 6b), while the apparent grain size increases for decreasing $\mathrm{C} / \mathrm{N}$ ratio. The nitrogen incorporation can be related to two structural modifications similar to those reported in a-C:H films $;{ }^{43}$ first, it increases the number and size of the $\mathrm{sp}^{2}$ bonds, also observed in the Raman measurements; second, it increases the density of voids. ${ }^{44}$ Both of these modifications can increase the surface roughness of the samples. ${ }^{45}$ The morphologic changes observed match the evolution of the CL emission obtained for different $[\mathrm{N}]$ added to the samples: the large roughness related with the incorporation of $\mathrm{N}$ reduces the luminescence emission, while the samples with smoother surfaces, corresponding to a low incorporation of $\mathrm{N}$, give a higher emission, and also better resolved luminescence bands, suggesting a much less distorted lattice with improved order. 

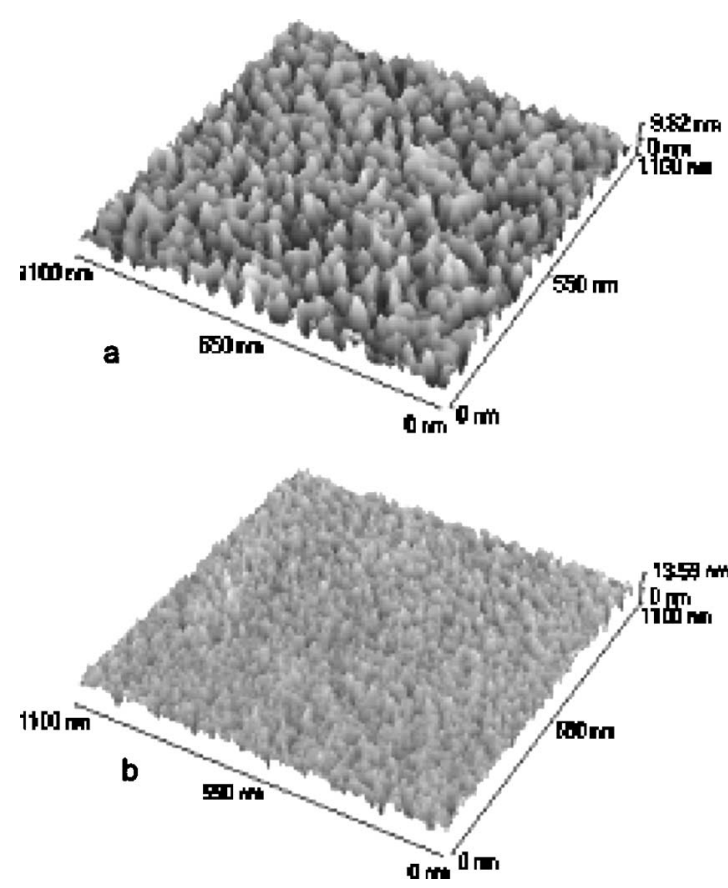

Figure 6. AFM images of samples deposited with $\mathrm{N} / \mathrm{Si}=0.8$ and (a) $\mathrm{C} / \mathrm{Si}=2.8($ series $\mathrm{A})$ and $(\mathrm{b}) \mathrm{C} / \mathrm{Si}=3.6($ series $\mathrm{B})$.

\section{Discussion}

The lack of graphitic phase obtained by ellipsometry can be related to the conductor nature of the graphitic bulk phase, whose dielectric constants were tried as a component for the EMA model in the spectral fittings. The typical strong absorption of the graphitic bulk phase over the whole spectral range of the ellipsometer is due to the nonhybridized p-orbital, partially occupied by the fourth electron of $\mathrm{C}$. Hence, this electron is not only responsible for the metallic behavior of graphite, but also for its optical properties. In our samples this behavior might be lost when this fourth electron is bonding with $\mathrm{Si}$ or $\mathrm{N}$.

As we have suggested previously, the shoulder contribution around $640 \mathrm{~cm}^{-1}$ cannot be assigned to $\mathrm{Si}-\mathrm{H}_{2}$ and $\mathrm{Si}-\mathrm{H}$ wagging bands because of the absence of the corresponding $\mathrm{Si}-\mathrm{H}$ stretching bands at around $2100 \mathrm{~cm}^{-1}$. However, the presence of $\mathrm{C}$ atoms in nearest-neighbor positions of the $\mathrm{Si}-\mathrm{Si}$ bonds, $\mathrm{Si}-\mathrm{Si}-\mathrm{C}$, could shift the $\mathrm{Si}-\mathrm{Si}$ vibration mode towards larger wavenumbers. In fact, similar bands have been observed around $664 \mathrm{~cm}^{-1}$ in amorphous $\mathrm{SiC}: \mathrm{H}^{26}$ The broad band in Raman spectra located at $640 \mathrm{~cm}^{-1}$ wavenumbers for the intermediate $\mathrm{C} / \mathrm{N}$ element flow ratios can be related to $\mathrm{Si}-\mathrm{Si} \mathrm{LA}$ vibrations. ${ }^{46}$

Concerning the broad Raman contributions labeled as I1 and I2 at 790 and $980 \mathrm{~cm}^{-1}$, respectively, it is observed that the relative intensity of both bands, I1/I2, is related to the composition of the samples (percentages of $\mathrm{SiC}$ and $\mathrm{Si}_{3} \mathrm{~N}_{4}$ ) obtained by ellipsometry: the band at $980 \mathrm{~cm}^{-1}$ increases its intensity with the $\mathrm{Si}_{3} \mathrm{~N}_{4}$ content, while the other band, $790 \mathrm{~cm}^{-1}$, follows the opposite evolution, which allows us to associate the $790 \mathrm{~cm}^{-1}$ band with the $\mathrm{SiC}$ bonds and the $980 \mathrm{~cm}^{-1}$ band with the $\mathrm{Si}-\mathrm{N}$ bonds. The broadness of these bands suggests a strong lattice distortion.

Nitrogen incorporation to the amorphous carbon to form amorphous $\mathrm{CN}$ films gives rise to the appearance of a band around $1500 \mathrm{~cm}^{-1}$ further than the usual D and G bands. ${ }^{35}$ However, the similarity of $\mathrm{C}-\mathrm{C}$ and $\mathrm{C}-\mathrm{N}$ vibrational frequencies makes it difficult to establish the way in which nitrogen is incorporated to the network skeletal. $^{36}$ The Raman spectra in the $1200-1600 \mathrm{~cm}^{-1}$ wavenumber range consists of a broad band peaking at $1500 \mathrm{~cm}^{-1}$ with a large asymmetry towards the low wavenumbers.
The Raman spectra in the $1200-1600 \mathrm{~cm}^{-1}$ spectral region for sample series A $(\mathrm{C} / \mathrm{Si}=2.8)$ are fitted in Fig. 7, following a twoGaussian scheme for the two bands G $\left(1580-1600 \mathrm{~cm}^{-1}\right)$ and D $\left(1360 \mathrm{~cm}^{-1}\right)$ that could be assigned to chainlike and ringlike $\mathrm{CN}$ molecules, respectively. ${ }^{36}$ The results show that the G band shifts to the low frequencies and the $I_{\mathrm{D}} / I_{\mathrm{G}}$ tends to zero when $[\mathrm{N}]$ decreases (C/N atomic flow ratio increases). For low $[\mathrm{N}]$ the Raman spectrum consists of a single broad band centered at $1430 \mathrm{~cm}^{-1}$, which can be assigned to $\mathrm{C}-\mathrm{C}$ or $\mathrm{C}-\mathrm{N}$ bonds in a structure that can be described as a covalent network of tetrahedral-trigonal bonding carbons (mixed $\mathrm{sp}^{2}-\mathrm{sp}^{3}$ hybridization) with distorted bond angles and bond lengths. ${ }^{47}$ In an opposed sense, it is possible to interpret the shift of the $\mathrm{G}$ band and the $I_{\mathrm{D}} / I_{\mathrm{G}}$ intensity ratio when $[\mathrm{N}]$ increases as the result of the increase in the $\mathrm{sp}^{2}$ bonding fraction and the bigger size of the disordered (graphitic) $\mathrm{sp}^{2}$ bonded clusters. ${ }^{48}$ According to the scenario described by the model of Ferrari et al., ${ }^{32}$ the observed evolution of the Raman spectra in this region can be interpreted using an amorphization trajectory from nanocrystalline material, either graphite or $\mathrm{CN}$, to a-C or a-CN when the $[\mathrm{N}]$ decreases. The assignment of $\mathrm{G}$ and $\mathrm{D}$ Raman bands to $\mathrm{C}-\mathrm{C}$ bonds cannot be completely ruled out; however, the rejection of graphite contribution in the ellipsometric spectra fittings and the absence of bands in the $2000 \mathrm{~cm}^{-1}$ in the FTIR spectra suggest that $\mathrm{C}-\mathrm{N}$ related bands should be dominant in the Raman spectra. In support of this assertion, the weak band around $1125 \mathrm{~cm}^{-1}$ observed in the FTIR spectra has been related to the $\mathrm{sp}^{3} \mathrm{C}-\mathrm{N}$ vibration. ${ }^{49}$

According to this, along with the main FTIR contributions related to the $\mathrm{Si}-\mathrm{N}$ and $\mathrm{Si}-\mathrm{C}$ modes, one could argue that $\mathrm{SiCN}$ films should consist of Si-centered tetrahedra, bonded to N, C, and Si, as the fundamental structural units, $\mathrm{SiC}_{n} \mathrm{~N}_{4-n}$, with ringlike and chainlike bridging $\mathrm{CN}$ structures.

\section{Conclusions}

Hydrogen-free SiCN films have been obtained by ECR plasma using a wide range of precursor gas flow conditions. Films mainly consist of $\mathrm{Si}-\mathrm{C}$ and $\mathrm{Si}-\mathrm{N}$ bonds, whose relative abundances follow the $\mathrm{C} / \mathrm{N}$ atomic ratio variation of the precursor gases. When this ratio increases, $\mathrm{Si}-\mathrm{Si}$ bonding becomes more evident. $\mathrm{C}-\mathrm{C}$ and $\mathrm{C}-\mathrm{N}$ bonds are also detected, in $\mathrm{sp}^{2}$ and $\mathrm{sp}^{3}$ hybridizations, with relative concentrations also depending on the $\mathrm{C} / \mathrm{N}$ ratio. Low $\mathrm{C} / \mathrm{N}$ values favor the presence of both $\mathrm{sp}^{3}$ and $\mathrm{sp}^{2}$ (graphitic) bonds, while in the opposite case an amorphous $\mathrm{C}$ or $\mathrm{CN}$ phase related to the graphitic $\mathrm{sp}^{2}$ bonding gradually becomes the only contribution to the Raman band.

In accordance with the EMA theory, the optical responses of these films in the UV-visible spectral range correspond to those of compounds with composition between $\mathrm{Si}_{3} \mathrm{~N}_{4}$ and $\mathrm{SiC}$. In the case of the films with higher $\mathrm{C} / \mathrm{N}$ ratios an additional $\mathrm{c}$-Si contribution gives rise to more absorbent films.

The absence of $\mathrm{H}$ in these films allows us to expect optimal mechanical properties and therefore for them to be appropriate for MEMS technology. Together with the mechanical goodness, the chemical stability, characteristic of $\mathrm{Si}-\mathrm{C}, \mathrm{Si}-\mathrm{N}, \mathrm{C}-\mathrm{C}$, and $\mathrm{C}-\mathrm{N}$ bonds, can be predicted for the so-obtained carbonitride compounds, which should make them suitable to be used in aggressive environments.

The luminescence emission of the films was optimized to increase the $\mathrm{SiC}$ abundance.

\section{Acknowledgment}

This work has been supported by the Spanish MCYT MAT200204494-C02 project.

University of Valladolid assisted in meeting the publication costs of this article. 

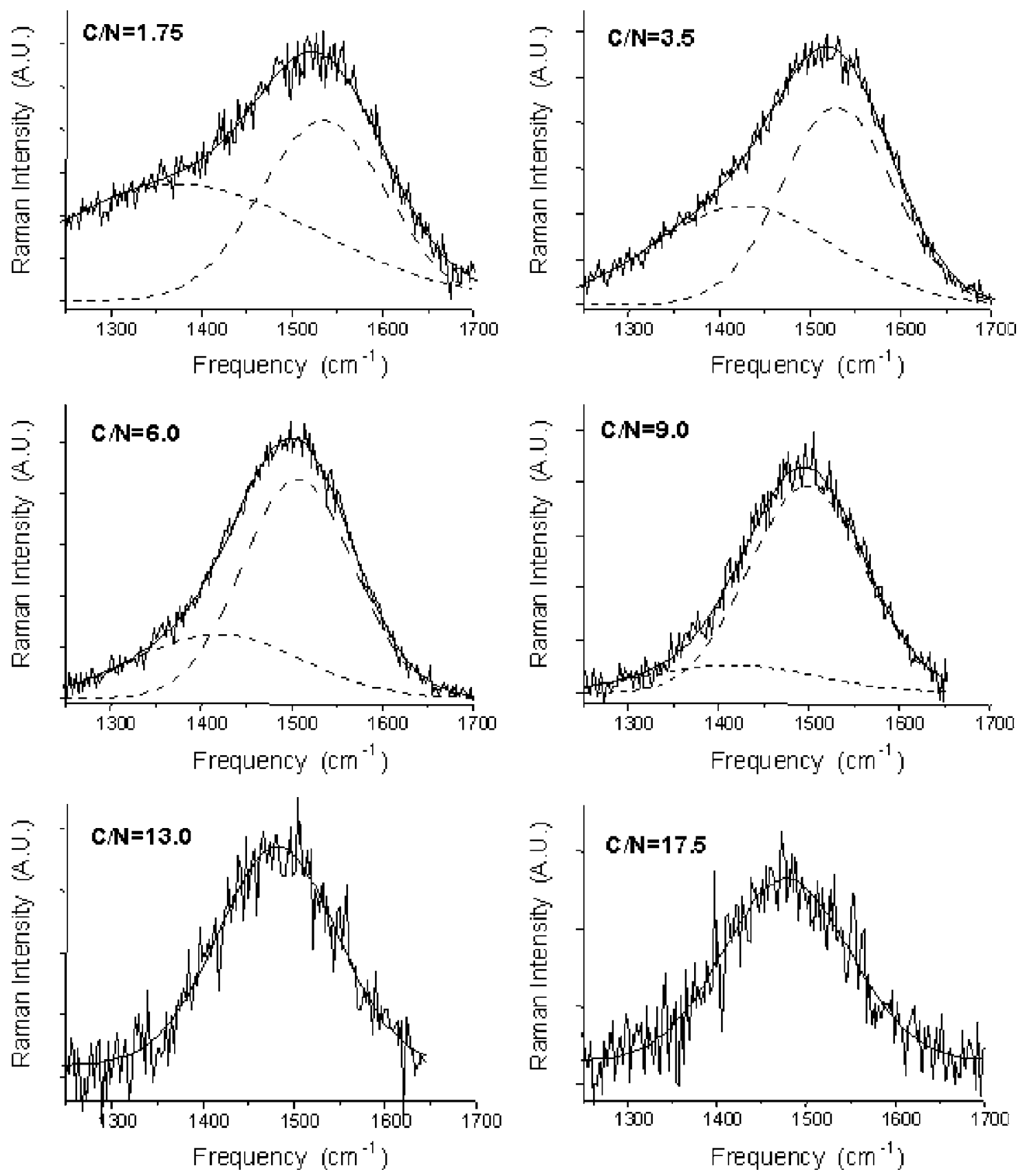

Figure 7. Fitting of Raman spectra bands at the 1200-1600 region with Gaussian peaks.

\section{References}

1. R. Riedel, H. Kleebe, H. Schonfelder, and F. Aldinger, Nature (London), 374, 526 (1995).

2. A. Bendeddouche, R. Berjoan, E. Beche, and R. Hillel, Surf. Coat. Technol., 111, 184 (1999).

3. S. Miyagawa, K. Baba, M. Ikeyama, K. Saitoh, S. Nakao, and Y. Miyagawa, Nucl. Instrum. Methods Phys. Res. B, 127-128, 651 (1997).

4. L. Barbadillo, M. J. Hernández, M. Cervera, P. Rodríguez, J. Piqueras, S. I. Molina, F. M. Morales, and D. Araujo, Nucl. Instrum. Methods Phys. Res. B, 184, 361 (2001).

5. A. Bendeddouche, R. Berjoan, E. Beche, T. Merle-Mejean, S. Schamm, V. Serin, G. Taillades, A. Pradel, and R. Hillel, J. Appl. Phys., 81, 6147 (1997).

6. L. C. Chen, C. K. Chen, S. L. Wei, D. M. Bushari, K. H. Chen, Y. F. Chen, Y. C. Jong, and Y. S. Huang, Appl. Phys. Lett., 72, 2463 (1998).

7. L. Barbadillo, F. J. Gómez, M. J. Hernández, and J. Piqueras, Appl. Phys. A: Mater. Sci. Process., 68, 1 (1999)

8. H. C. Lo, J. J. Wu, C. Y. Wen, T. S. Wong, S. T. Lin, K. H. Chen, and L. C. Chen, Diamond Relat. Mater, 10, 1916 (2001).

9. L. C. Chen, S. W. Chang, C. S. Chang, C. Y. Wen, J.-J. Wu, Y. F. Chen, Y. S. Huang, and K. H. Chen, J. Phys. Chem. Solids, 62, 1567 (2001).
10. H. L. Chang, C. M. Hsu, and C. T. Kuo, Appl. Phys. Lett., 80, 4638 (2002).

11. S.-F. Ting, Y.-K. Fang, W.-T. Hsieh, Y.-S. Tsair, C.-N. Chang, C.-S. Lin, M.-C. Hsieh, H.-C. Chiang, and J.-J. Ho, IEEE Electron Device Lett., 23, 142 (2002).

12. I. Martin, M. Vetter, A. Orpella, C. Voz, J. Puigdollers, and R. Alcubilla, Appl. Phys. Lett., 81, 4461 (2002).

13. C. W. Chen, M.-H. Lee, L. C. Chen, and K. H. Chen, Diamond Relat. Mater, 13, 1158 (2004).

14. P. Jedrzejowski, J. Cizek, A. Amassian, J. E. Klemberg-Sapieha, J. Vlcek, and L. Martinu, Thin Solid Films, 447-448, 201 (2004).

15. W.-R. Chang, Y.-K. Fang, S.-F. Ting, Y.-S. Tsair, C.-N. Chang, C.-Y. Lin, and S.-F. Chen, IEEE Electron Device Lett., 24, 565 (2003).

16. W.-R. Chang, Y.-K. Fang, S.-F. Ting, S.-F. Chen, C.-Y. Lin, S.-B. Hwang, and C.-N. Chang, J. Electron. Mater, 33, 181 (2004).

17. H. Misaki, M. Asano, K. Kasai, M. Fukuda, and N. Imamura, IEEE Trans. Magn., 25, 4030 (1989).

18. F. J. Gómez, P. Prieto, E. Elizalde, and J. Piqueras, Appl. Phys. Lett., 69, 773 (1996)

19. F. G. Tantair, C. Y. Wen, L. C. Chen, J.-J. Wu, K. H. Chen, P. F. Kuo, S. W. Chang, Y. F. Chen, W. K. Hong, and H. C. Chen, Appl. Phys. Lett., 76, 2630 (2000).

20. Y. P. Liu, L. A. Liew, R. L. Luo, L. N. An, M. L. Dunn, V. M. Bright, J. W. Daily, 
and R. Raj, Sens. Actuators, A, 95, 120 (2002).

1. L. A. Liew, R. A. Saravanan, V. M. Bright, M. L. Dunn, J. W. Daily, and R. Raj, Sens. Actuators, A, 103, 171 (2003)

22. H. Cui and P. A. Burke, J. Electrochem. Soc., 151, G795 (2004).

23. G. Spitzer, D. A. Kleiman, and D. Walsh, Phys. Rev., 113, 127 (1959).

24. D. R. Lee, G. Lucovsky, M. S. Den, and Ch. Magee, J. Vac. Sci. Technol. A, 13, 1671 (1995).

25. J. H. Kaufman, S. Metin, and D. D. Saperstein, Phys. Rev. B, 39, 13053 (1989).

26. V. P. Tolstoy, I. V. Chernyshova, and V. A. Skryshevsky, in Handbook of Infrared Spectroscopy of Ultrathin Films, pp. 445-452, John Wiley \& Sons, New York (2003).

27. Y. Sasaki, Y. Nishima, M. Sato, and K. Okamura, Phys. Rev. B, 40, 1762 (1989).

28. N. I. Fainer, Yu. M. Rumyantsev, A. N. Golubenko, M. L. Kosinova, and F. A. Kuznetsov, J. Cryst. Growth, 248, 175 (2003).

29. I. V. Afanasyev-Charkin, L. G. Jacobsohn, R. D. Averitt, and M. Nastasi, J. Vac. Sci. Technol. A, 22, 2342 (2004)

30. F. Tuistra and S. A. Solin, J. Chem. Phys., 53, 1126 (1970).

31. C. Chieu, G. Dresselhaus, and M. Endo, Phys. Rev. B, 26, 5867 (1982).

32. A. C. Ferrari and J. Robertson, Phys. Rev. B, 61, 14095 (2000).

33. Y. F. Lu, Z. F. He, Z. H. Mai, and Z. M. Ren, J. Appl. Phys., 88, 7095 (2000).

34. G. Messina, S. Santangelo, G. Fanchini, A. Tagliaferro, C. E. Nobili, G. Ottaviani, A. Paoletti, and A. Tucciarione, Diamond Relat. Mater., 11, 1166 (2002).

35. Z. Qin, P. Wang, H. Shen, L. Mi, and X. Ying, Diamond Relat. Mater, 14, 1616 (2005).

36. A. C. Ferrari, S. E. Rodil, and J. Robertson, Phys. Rev. B, 67, 155306 (2003)
37. Y. Ward, R. J. Young, and R. A. Shatwell, Composites, Part A, 33, 1409 (2002)

38. D. E. Aspnes, in Selected Papers on Ellipsometry, pp 312-319, SPIE Milestone Series, MS 27, R. M. A. Azzam and B. J. Thompson, Editors, SPIE Optical Engineering Press, Bellingham, WA (1991).

39. D. E. Aspnes and A. A. Studna, Phys. Rev. B, 27, 985 (1983).

40. Y. Shi, Y. Xin, Z. X. Huang, L. Pu, R. Zhang, and Y. D. Zheng, Physica E (Amsterdam), 30, 41 (2005).

41. Y. H. Wang, J. Lin, and C. H. A. Huan, Mater. Sci. Eng., B, 95, 43 (2002).

42. T. P. Smirnova, A. M. Badalian, L. V. Yakovkina, V. V. Kaichev, V. I. Bukhtiyarov, A. M. Shmakov, I. P. Asanov, V. I. Rachlin, and A. N. Fomina, Thin Solid Films, 429, 144 (2003).

43. G. Mariotto, F. L. Freire, Jr., and C. A. Achete, Thin Solid Films, 241, 255 (1994).

44. F. L. Freire, Jr., C. A. Achete, R. B. Brusa, G. Mariotto, X. T. Teng, and A. Zeeca, Solid State Commun., 91, 23 (1993).

45. D. H. Chen, A. X. Wei, S. P. Wong, J. B. Xu, M. M. Wu, and S. Q. Peng, Appl. Phys. A: Mater. Sci. Process., 70, 47 (2000).

46. Z. Hu, X. Liao, H. Diao, G. Kongh, X. Zeng, and Y. Xu, J. Cryst. Growth, 264, 7 (2004).

47. E. J. Liang, J. W. Zhang, J. Leme, C. Moura, and L. Cunha, Thin Solid Films, 469-470, 410 (2004).

48. L. Escobar Alarcón, J. E. Villareal-Barajas, E. Camps, S. Muhl, E. HaroPoniatowski, S. Romero, and B. Salinas, Mater. Sci. Eng., B, 90, 79 (2002).

49. X. C. Xiao, Y. W. Li, L. X. Song, X. F. Peng, and X. F. Hu, Appl. Surf. Sci., 156, $155(2000)$ 